\title{
General note on the theorem of Stampfli
}

\section{Cheniti Bensalloua and Mostefa Nadir}

*Correspondence: mostefanadir@yahoo.fr

Department of Mathematics, University of Msila, Msila, Algeria

\begin{abstract}
It is well known that for every bounded operator $A$ in $L(H)$, there exists a compact operator $K$ in $K(H)$ such that the Weyl spectrum $\sigma_{W}(A)$ of the operator $A$ coincides with the spectrum $\sigma(A+K)$ of the perturbed operator $A$. In this work, we show the extension of this relation by the use of Kato's decomposition to the set of semi-Fredholm operators.
\end{abstract}

MSC: Primary 05C38; 15A15; secondary 05A15; $15 \mathrm{~A} 18$

Keywords: semi-Fredholm operator; index of semi-Fredholm operator; Weyl spectrum

\section{Introduction}

In 1965 Schechter demonstrated in [1] this result that for every bounded operator $A$ in $L(H)$, there exists a compact operator $K$ in $K(H)$ such that the Weyl spectrum $\sigma_{W}(A)$ of the operator $A$ coincides with the spectrum $\sigma(A+K)$ of the perturbed operator $A$ for a compact perturbation of Fredholm operators of index zero. In 1973 Stampfli showed in [2] that the minimum of this perturbation is achieved for a certain compact operator. This work is intended to extend this result to semi-Fredholm operators of any index using the decomposition of Kato, an extension already done by Apostol in [3] and Herrero in [4].

Let $H$ be a complex, separable, infinite dimensional Hilbert space, and let $L(H)$ denote the algebra of all linear bounded operators on $H, C(H)$ the set of linear operators $A$ with domain $D(A)$ dense in $H$ and range $R(A)$ contained in $H$ and a graph $G(A)$ closed in $H \times H$. $K(H)$ is the set of compact elements of $L(H)$.

For $A \in C(H)$, we let $\sigma(A), \rho(A)$, and $N(A)$ denote the spectrum, the resolvent set, and the null space of $A$, respectively. The nullity $\alpha(A)$ of $A$ is defined as the dimension of $N(A)$ and the deficiency $\beta(A)$ of $A$ is defined as the codimension of $R(A)$ in $H$.

$\sigma_{W}(A)$ will denote the Weyl spectrum. Recall that the Weyl spectrum is the union of the essential spectrum $\sigma_{e}(A)$ and all bounded components of $\mathbb{C} / \sigma_{e}(A)$ associated with a nonzero Fredholm index. In other words, the Weyl spectrum of $A$ is the set

$$
\sigma_{W}(A)=\sigma(A) \backslash \Phi_{0}(A),
$$

where $\Phi_{0}(A)$ designates the set of all scalars $\mu \in \mathbb{C}$ such that $R(A-\mu I)$ is closed and both $\operatorname{dim} N(A-\mu I)$ and $\operatorname{dim} N\left(A^{*}-\bar{\mu} I\right)$ are finite such that the index $\operatorname{ind}(A-\mu I)$ of $(A-\mu I)$ is null where

$$
\operatorname{ind}(A-\mu I)=\operatorname{dim} N(A-\mu I)-\operatorname{dim} N\left(A^{*}-\bar{\mu} I\right) .
$$

(C) 2016 Bensalloua and Nadir. This article is distributed under the terms of the Creative Commons Attribution 4.0 International License (http://creativecommons.org/licenses/by/4.0/), which permits unrestricted use, distribution, and reproduction in any medium, provided you give appropriate credit to the original author(s) and the source, provide a link to the Creative Commons license, and indicate if changes were made. 
Note that it was shown in [1] that for every bounded operator $A$ in $L(H)$, there exists a compact operator $K$ in $K(H)$ such that

$$
\sigma_{W}(A)=\sigma(A+K) .
$$

Proposition 1 (See [5]) For $A \in C(H)$ we get

(1) $R(A)$ is a closed subspace in $H$ if and only if $c(A)>0$,

(2) $c(A)=c\left(A^{*}\right)$,

where $A^{*}$ is the adjoint of $A$ and $c(A)$ is given by

$$
c(A)=\inf _{u \in D(A) \cap N(A)^{\perp}} \frac{\|A u\|}{\|u\|} .
$$

Definition 1 The operator $A \in C(H)$ is called a semi-Fredholm operator, denoted $A \in$ $S F(H)$, if the following conditions are satisfied:

(1) $R(A)$ is a closed subspace in $H$,

(2) $\min \operatorname{ind}(A)=\left\{\operatorname{dim} N(A), \operatorname{dim} N\left(A^{*}\right)\right\}<\infty$.

Theorem 1 (See [6]) Let $A \in S F(H)$, there is a direct decomposition $H=M \oplus N$ such that

(1) $M$ and $N$ are invariant by $A$,

(2) $A /_{M}$ is regular,

(3) $N \subseteq D(A), \operatorname{dim} N<\infty$, and $A / N$ is nilpotent.

This decomposition is known as the decomposition of Kato [6]. Operators admitting such decomposition were characterized in 1976 by Labrousse in [7] and are called quasi-Fredholm operators, also generalized by Mbekhta to the operators called pseudoFredholm [8].

Definition 2 Let $A \in C(H)$; the complex set $\rho_{e}(A)$ of the operator $A$ given by

$$
\rho_{e}(A)=\{\mu \in \mathbb{C},(A-\mu I) \in S F(H)\}
$$

will be called the essential resolvent (resp. $\sigma_{e}(A)=\mathbb{C} \backslash \rho_{e}(A)$ ) and it will stand for the essential spectrum of the operator $A$.

For $A \in C(H)$, we define the set

$$
M_{e}(A)=\{\mu \in \mathbb{C} \text { such that }(A-\mu I) \text { is invertible on the left or on the right }\} .
$$

Lemma 1 If $(A-\mu I) \in S F(A)$ such that

$$
\min \left\{\operatorname{dim} N(A-\mu I), \operatorname{dim}\left(A^{*}-\mu I\right)\right\}=0,
$$

then $\mu \in M_{e}(A)$.

Definition 3 Let $A \in C(H)$, the complex point $\mu \in \mathbb{C}$ is called a regular point of $A$, denoted $\operatorname{reg}(A)$, if the following conditions are satisfied: 
(1) $R(A-\mu I)$ is a closed subspace in $H$,

(2) $N\left[(A-\mu I)^{n}\right] \subseteq R(A-\mu I), \forall n \in \mathbb{N}$.

Remark 1 The set $\operatorname{reg}(A)$ of regular points of $A$ is open in $\mathbb{C}$.

Theorem 2 (See [9]) $A \in S F(H)$ if and only if $A^{*} \in S F(H)$ and $\operatorname{ind}(A)=-\operatorname{ind}\left(A^{*}\right)$.

Theorem 3 (See [10]) For any $A \in L(H) \cap S F(H)$ and $B \in L(H)$ such that $(A-B) \in K(H)$ we get $B \in S F(H)$ and $\operatorname{ind}(A)=\operatorname{ind}(B)$.

Theorem 4 (See $[11])$ ind $(A-\mu I)$ is constant on each connected component of $\rho_{e}(A)$.

Definition 4 Let $A, B \in C(H)$; we denote by $P_{G(A)}$ the orthogonal projection of $H \times H$ on $G(A)$. We set

$$
\delta(A, B)=\left\|\left(I-P_{A}\right) P_{B}\right\|
$$

and

$$
g(A, B)=\left\|P_{G(A)}-P_{G(B)}\right\| .
$$

Remark $2 g(A, B)$ defines a metric on $C(H)$.

Proposition 2 (See [5]) Let $M, N$ be two closed subspaces of $H$ with $\operatorname{dim} N<\infty$. Then if $\delta(M, N)<1$ we have $\operatorname{dim} M \leq \operatorname{dim} N$.

Definition 5 Let $A \in L(H)$. $A$ is called quasi-nilpotent and will be noted $A \in Q N(H)$ if and only if $\lim _{n \rightarrow \infty}\left\|A^{n}\right\|^{\frac{1}{n}}=0$.

Proposition 3 (See [12]) Let $A \in L(H)$, then $A \in Q N(H)$ if and only if $\sigma(A)=0$.

Definition $6 A \in L(H)$ is called a Riesz operator if and only if $\sigma_{e}(A)=0$.

Theorem 5 (See [1]) Let $A \in L(H)$, that is, $K \in K(H)$ such that

$$
\sigma_{W}(A)=\bigcap_{K \in K(H)} \sigma(A+K) .
$$

In 1973 Stampfli demonstrated in [2] that there is an operator $K \in K(H)$ such that

$$
\sigma_{W}(A)=\sigma(A+K)
$$

Passing to the complement in the expression $\sigma_{W}(A)=\sigma(A+K)=\sigma(A) \cap\left[\Phi_{0}(A)\right]^{c}$ we obtain $\rho(A) \cup \Phi_{0}(A)=\rho(A+K)$. Hence $\rho(A) \subseteq \Phi_{0}(A)$; then the result of Stampfli is equivalent to $\Phi_{0}(A)=\rho(A+K)$.

\section{Main results}

Theorem 6 Let $A \in L(H)$, there exists a compact operator $K \in K(H)$ such that

$$
\rho_{e}(A)=M_{e}(A+K)
$$




\subsection{Correction of the operator $A$}

\subsubsection{First correction}

This correction involves the construction of a compact perturbation $K_{1}$ such that if $A \in$ $L(H)$ and $B=\left(A+K_{1}\right) \in L(H)$ then

$$
\rho_{e}(A) \cap \operatorname{reg}\left(A+K_{1}\right) \subseteq M_{e}\left(A+K_{1}\right)
$$

Lemma 2 Let $A \in L(H)$ such that $A$ is invertible on the left or on the right, $K_{1}$ an operator of $K(H)$ and $B=A+K_{1}$. If $\left\|K_{1}\right\|<c(A)$ then

(1) $\delta(N(B), N(A)) \leq \frac{\left\|K_{1}\right\|}{c(A)}$

(2) $B$ is invertible on the left or on the right.

Proof We have by definition $\delta(N(B), N(A))=\left\|\left(I-P_{N(B)}\right) P_{N(A)}\right\|$. Assume that $u \in H$ and let $v=\left(I-P_{N(A)}\right) P_{N(B)} u$. Then $v$ is orthogonal to $N(A)$ and we have

$$
\|A v\| \geq c(A)\|v\|
$$

or still

$$
\begin{aligned}
\|v\| \leq & \frac{\|A v\|}{c(A)}, \\
\frac{\|A v\|}{c(A)} & =\frac{\left\|A\left(\left(I-P_{N(A)}\right) P_{N(B)} u\right)\right\|}{c(A)} \\
& =\frac{\left\|A\left(P_{N(B)} u\right)\right\|}{c(A)} \\
& =\frac{\left\|(A-B)\left(P_{N(B)} u\right)\right\|}{c(A)} \\
& \leq \frac{\|(A-B)\|\left\|P_{N(B)} u\right\|}{c(A)} .
\end{aligned}
$$

Using Proposition 2, we have

$$
\delta(N(B), N(A))<1 \Rightarrow \operatorname{dim} N(B) \leq \operatorname{dim} N(A) .
$$

If $A$ is one-to-one, then $\operatorname{dim} N(A)=0 \Rightarrow \operatorname{dim} N(B)=0$. Therefore $N(B)=\{0\}$, hence $B$ is one-to-one. As $R(B)$ is closed (compact perturbation of semi-Fredholm operator), we deduce that $B$ is left invertible.

If $N(A) \neq\{0\}$, then $A$ is not left invertible. Hence $A$ is right invertible. Then $N\left(A^{*}\right)=\{0\}$. $A, B \in L(H) \Rightarrow A^{*}, B^{*} \in L(H), A^{*}-B^{*}=A-B$, and $c(A)=c\left(A^{*}\right)$.

By a similar reasoning we will have

$$
\delta\left(N\left(B^{*}\right), N\left(A^{*}\right)\right) \leq \frac{\left\|A^{*}-B^{*}\right\|}{c\left(A^{*}\right)}<1 \Rightarrow \operatorname{dim} N\left(B^{*}\right)=0 .
$$

Then $N\left(B^{*}\right)=\{0\}$ and $B^{*}$ is one-to-one. $B=\left(A+K_{1}\right) \in S F(H)$, in particular $R(B)$ is a closed subspace of $H, R(B)=N\left(B^{*}\right)^{\perp}=\{0\}^{\perp}=H$, then $B$ is onto. Thus it is right invertible. 
Lemma 3 Let $M, N$ be two closed subspace of $H$ such that $\operatorname{dim} N \geq \operatorname{dim} M>0$ and $\operatorname{dim} M<$ $\infty$. Then there is $U$, a partial isometry of $H$ such that

$$
R(U) \subseteq N \text { and } R\left(U^{*}\right)=M .
$$

Proof Let $e_{1}, e_{2}, \ldots, e_{m}$ be an orthonormal bases of $M$ and $f_{1}, f_{2}, \ldots, f_{m}$ an orthonormal family of vectors of $N$. Let $P$ the orthogonal projection of $H$ onto $M$ and $Q$ the orthogonal projection onto the space spanned by $f_{1}, f_{2}, \ldots, f_{m}$. If $u \in H$, there are $m$ complex numbers $a_{1}, a_{2}, \ldots, a_{m}$ such that $P u=\sum_{i=1}^{m} a_{i} e_{i}$. We put $U u=\sum_{i=1}^{m} a_{i} f_{i}$. Then $R(U) \subseteq N$ and if $v \in H$, there are $m$ complex numbers $b_{1}, b_{2}, \ldots, b_{m}$ such that $Q v=\sum_{i=1}^{m} b_{i} f_{i}$. Then $\langle U u, v\rangle=\sum_{i=1}^{m} a_{i} \bar{b}_{i}=\left\langle u, \sum_{i=1}^{m} b_{i} e_{i}\right\rangle=\left\langle u, U^{*} v\right\rangle$, hence $U^{*} v=\sum_{i=1}^{m} b_{i} e_{i}$. It is easy to see that $R(U *)=M, U$ is a partial isometry of $H$, and

$$
U^{*} U=P, \quad U U^{*}=Q
$$

Lemma 4 Let $A \in L(H), \beta \in \rho_{e}(A)$, and $\alpha \in \mathbb{R}_{+}^{*}$. Then there is $T(A, \beta) \in L(H)$ such that

(1) $T(A, \beta)$ is of finite rank,

(2) $\|T(A, \beta)\| \leq 1$,

(3) $\beta \in M_{e}(A+\alpha T(A, \beta))$.

Proof We suppose at first ind $(A-\beta I) \geq 0$. Hence $\operatorname{dim} N\left(A^{*}-\beta I\right)<\infty$. By Lemma 3 , there is $U$ such that $R(U) \subseteq N(A-\beta I), R\left(U^{*}\right)=N\left(A^{*}-\beta I\right)$. We take $U^{*}=T(A, \beta)$. Then $T(A, \beta)$ is of finite rank, thus compact, and we have $\beta \in \rho_{e}(A+\alpha T(A, \beta))$. Furthermore $A+\alpha T(A, \beta)-$ $\beta I$ is onto. Indeed $R(A-\beta I)$ is a closed subspace of $H$. Hence $H=R(A-\beta I) \oplus N\left(A^{*}-\beta I\right)$. Let $u \in H$, then $u=u_{1}+u_{2}$ with $u_{1} \in R(A-\beta I)$ and $u_{2} \in N(A *-\beta I)$. Let $Q$ be a projection onto $N(A-\beta I)$. There is $v_{1}$ and $v_{2}$ such that $(A-\beta I) v_{1}=u_{1}$ where $Q v_{1}=0$ and $u_{2}=\alpha T v_{2}$, where $v_{2}=Q v_{2}$, that is, $v_{2} \in N(A-\beta I)$. If we take $v=v_{1}+v_{2}$ it follows that

$$
\begin{aligned}
(A+\alpha T(A, \beta)-\beta I) v & =(A-\beta I)(I-Q) v_{1}+\alpha T(A, \beta) v_{2} \\
& =(A-\beta I) v_{1}+\alpha T(A, \beta) v_{2}=u_{1}+u_{2}=u .
\end{aligned}
$$

Hence the operator $(A+\alpha T(A, \beta)-\beta I)$ is onto and $\beta \in M_{e}(A+\alpha(T, \beta))$. If ind $(A-\beta I)<0$ and $\operatorname{dim} N(A-\beta I)>0$, we proceed as above by replacing $A-\beta I$ by $A^{*}-\beta I$ and $T$ by $T^{*}$ as $\operatorname{ind}(A-\beta I)=-\operatorname{ind}\left(A^{*}-\beta I\right)$. The operator $(A+\alpha T(A, \beta)-\beta I)$ is one-to-one, it follows that

$$
\beta \in M_{e}(A+\alpha T(A, \beta))
$$

Remark 3 If $\operatorname{ind}(A-\beta I)=0$, then $A+\alpha T(A, \beta)-\beta I$ is onto and one-to-one.

Remark 4 If $\min \operatorname{ind}(A-\beta I)=0$, we take $T=0$. Indeed in this case $A+\alpha T(A, \beta)-\beta I=$ $A-\beta I$ is invertible on the left or right.

Lemma 5 Let $A \in L(H)$. Then for all $\varepsilon>0$, there is $K_{1} \in K(H)$ such that

(1) $\left\|K_{1}\right\| \leq \varepsilon$,

(2) $\rho_{e}\left(A+K_{1}\right) \cap \operatorname{reg}\left(A+K_{1}\right) \subseteq M_{e}\left(A+K_{1}\right)$. 
Proof Suppose that $\rho_{e}(A)=\rho_{e}(B)=\bigcup_{1 \leq i \leq n} C_{i}$ where $C_{i}, i=1,2,3, \ldots, n$, is a connected component. For each $i$, let $\beta_{i} \in C_{i}$. We inductively define a sequence of positive real numbers and a sequence of operators in $L(H)$ as follows:

(1) $\alpha_{1}=\alpha, A_{0}=A$,

(2) $A_{i}=A_{i-1}+\alpha_{i} T_{i}, i=1,2,3, \ldots, n$, where $T_{i}=T\left(A_{i-1}, \beta_{i}\right)$,

(2) $\alpha_{i+1}=\frac{1}{3} \min \left\{\alpha_{i}, c\left(A_{i}-\beta_{i} I\right), i=1,2,3, \ldots, n-1\right\}$.

Note that (1) and (3) give $\alpha_{i+1} \leq \frac{1}{3} \alpha_{i} \Rightarrow \alpha_{i+1} \leq \frac{\alpha}{3^{i}}$. We set $S_{i}=\sum_{1 \leq j \leq i} \alpha_{j} T_{j}$; then

$$
\left\|S_{i}\right\| \leq \sum_{1 \leq j \leq i} \alpha_{j}\left\|T_{j}\right\| \leq \sum_{1 \leq j \leq i} \alpha_{j} \leq \sum_{1 \leq j \leq \infty} \alpha_{j} \leq \alpha \sum_{1 \leq j \leq \infty} \frac{1}{3^{j-1}}=\frac{3}{2} \alpha
$$

Therefore $\left\|S_{i}\right\| \leq \frac{3}{2} \alpha$. For each $i, A_{i}=A+S_{i}$ where $S_{i}$ is a finite rank operator and therefore compact. We must have $\rho_{e}(A)=\rho_{e}\left(A_{i}\right)$ and $c\left(A_{i}-\beta_{i} I\right)>0$. This shows that all $\alpha_{i}$ are strictly positive. In addition, by the previous lemma $A_{i}-\beta_{i} I$ is left or right invertible, from which we deduce that $C_{i} \cap \operatorname{reg}\left(A_{i}\right) \subseteq M_{e}\left(A_{i}\right)$. Finally

$$
\left\|S_{n}-S_{i}\right\| \leq \sum_{i+1 \leq j \leq n} \alpha_{j} \leq \frac{3}{2} \alpha_{i+1} \leq \frac{1}{2} c\left(A_{i}-\beta_{i} I\right)<c\left(A_{i}-\beta_{i} I\right) .
$$

Hence $A_{n}-\beta_{i} I-\left(A_{i}-\beta_{i} I\right)=S_{n}-S_{i}$; using Lemma 2, we deduce that for $i=1,2,3, \ldots, n$, $A_{n}-\beta_{i} I$ is left or right invertible and therefore $\rho_{e}\left(A+S_{n}\right) \cap \operatorname{reg}\left(A+S_{n}\right) \subseteq M_{e}\left(A+S_{n}\right)$. Accordingly we get $K_{1}=S_{n}$ and $\alpha=\frac{2}{3} \varepsilon$, and the theorem is proved. If $\rho_{e}(A)$ has a countable infinite numbers of connected components, the only other possible case, we observe that the sequence $\left(S_{n}\right)$ converges normally, and taking $K_{1}=\lim _{n \rightarrow \infty} S_{n}$, we proceed exactly as in the previous case. As, for every $n, S_{n}$ is an operator of finite rank, $K_{1}$ is a compact operator. We set $B=A+K_{1}$.

\subsubsection{Second correction}

Let $B \in L(H)$ such that $\rho_{e}(B) \cap \operatorname{reg}(B) \subseteq M_{e}(B)$. We will build a second compact perturbation $K_{2}$ such that $\rho_{e}(B) \subseteq M_{e}\left(B+K_{2}\right)$. If $B \in L(H)$ such that $\rho_{e}(B) \cap \operatorname{reg}(B) \subseteq M_{e}(B)$, then $\rho_{e}(B) \backslash M_{e}(B)$ is a finite or countable set denoted $\mu_{j}$. Note that $\sigma_{e}(B)=\emptyset$, because in [6], there exist $n \in \mathbb{N}$ and $\mu_{1}, \mu_{2}, \mu_{3}, \ldots, \mu_{n} \in \mathbb{C}$ such that $\sigma(B)=\left\{\mu_{1}, \mu_{2}, \mu_{3}, \ldots, \mu_{n}\right\}$. Hence $\operatorname{dim} H<\infty$, this is contrary to our hypotheses. As $\sigma_{e}(B)$ is a closed set of $\sigma(B)$ (which is compact), $\sigma_{e}(B)$ is also compact. Since the distance function is continuous on $\sigma_{e}(B)$, there is a sequence of points $\left(\mu_{l}^{\prime}\right)_{j \in \mathbb{N}^{*}} \in \sigma_{e}(B)$ such that $d\left(\mu_{j}, \sigma_{e}(B)\right)=\left|\mu_{j}-\mu_{j}^{\prime}\right|$. Finally if the sequence $\left(\mu_{n}\right)$ is infinite, then $\lim _{j \rightarrow \infty}\left|\mu_{j}-\mu_{j}^{\prime}\right|=0$, because otherwise $\mu_{n}$ would have an accumulation point in $\rho_{e}(B)$, which is contradictory. For every $\mu_{j}$, let $M_{j}, N_{j}$ be the two subspaces of $H$ corresponding to the decomposition of Kato of the operator $B-\mu_{j} I$ and let $P_{j}$ be the associated projection of the kernel $M_{j}$ and the range $N_{j}$.

\section{Construction of subspaces $M_{j}^{\prime}$ and $N_{j}^{\prime}$}

For $j \in \mathbb{N}$, let $M_{0}^{\prime}=H, M_{j+1}^{\prime}=M_{j}^{\prime} \cap M_{j+1}$, and $N_{1}^{\prime}=M_{1}^{\perp}, N_{j+1}^{\prime}=M_{j}^{\prime} \cap M_{j+1}^{\prime \perp}$. Finally, we denote by $P_{j}^{\prime}$ the orthogonal projection onto $N_{j}^{\prime}$.

\section{Lemma 6}

(1) If $1 \leq i \leq n, 1 \leq j \leq n$, and $i \neq j$ then $N_{i}^{\prime} \perp N_{j}^{\prime}$ if and only if $P_{i}^{\prime} P_{j}^{\prime}=0$. 
(2) If $1 \leq j \leq n$, then $N_{j}^{\prime} \oplus M_{j}^{\prime}=N_{j} \oplus M_{j}^{\prime}=M_{j-1}^{\prime}$ and $\operatorname{dim} N_{j}^{\prime}=\operatorname{dim} N_{j}<\infty$.

(3) If $1 \leq j \leq n$, then $\bigcap_{1 \leq i \leq j} N_{i}^{\prime}=M_{j}^{\prime}$ and $\operatorname{codim} M_{j}^{\prime}=\sum_{1 \leq i \leq j} \operatorname{dim} N_{i}$.

Proof For any $i>j$, we have $N_{i}^{\prime} \perp N_{j}^{\prime}$. Indeed $N_{i}^{\prime} \subseteq M_{i-1}^{\prime} \subseteq M_{j}^{\prime}$; and $N_{j}^{\prime} \subseteq M_{j}^{\prime \perp}$, then $M_{j}^{\prime} \subseteq$ $N_{j}^{\prime \perp}$ and we deduce $N_{i}^{\prime} \subseteq N_{j}^{\prime \perp}$. Suppose $P_{i}^{\prime} P_{j}^{\prime}=0$ and let $x \in N_{i}^{\prime}$. Then $\langle x, y\rangle=\left\langle P_{i}^{\prime} x, P_{j}^{\prime} y\right\rangle=$ $\left\langle x, P_{i}^{\prime} P_{j}^{\prime} y\right\rangle=0 . N_{i}^{\prime}$ is orthogonal to $N_{j}^{\prime}$. Reciprocally if $N_{i}^{\prime}$ is orthogonal to $N_{j}^{\prime}$ and $u \in H$, then $P_{j}^{\prime} u \in N_{j}^{\prime}$ and $P_{j}^{\prime} u \in N_{j}^{\prime \perp}$ then $P_{i}^{\prime} P_{j}^{\prime} u=0$ and $P_{i}^{\prime} P_{j}^{\prime}=0$.

$N_{j}^{\prime} \cap M_{j}^{\prime} \subseteq M_{j}^{\prime \perp} \cap M_{j}^{\prime}=\{0\}$ and $N_{j}^{\prime} \cap M_{j}^{\prime} \subseteq N_{j}^{\prime} \cap M_{j}^{\prime}=\{0\} . M_{j}^{\prime}+N_{j}^{\prime}=M_{j-1}^{\prime}$ (because $N_{j}^{\prime}=$ $M_{j-1}^{\prime} \cap M_{j}^{\prime \perp}$.

For any $i>j, N_{i}^{\prime} \subseteq M_{j-1}^{\prime}=N_{j}^{\prime} \oplus M_{j}^{\prime}$, then $M_{j}^{\prime} \oplus N_{j}^{\prime} \subseteq M_{j}^{\prime} \oplus N_{j}^{\prime} ; N_{j}^{\prime} \subseteq M_{j-1}^{\prime}=M_{j}^{\prime} \oplus N_{j} \Rightarrow$ $M_{j}^{\prime} \oplus N_{j}^{\prime} \subseteq M_{j}^{\prime} \oplus N_{j}$.

If $i=j$, the result is obvious. Suppose that it is true for $j$. Then $\operatorname{codim} M_{j}^{\prime}<\infty$ and therefore $M_{j}^{\prime \perp} \oplus M_{j+1}^{\prime}$ is closed. Hence $M_{j}^{\prime \perp} \oplus M_{j+1}^{\prime}=N_{j+1}^{\prime \perp}$ and we have

$$
\bigcap_{1 \leq i \leq j+1} N_{i}^{\prime \perp}=M_{j}^{\prime} \cap N_{j+1}^{\prime \perp}=M_{j}^{\prime} \cap\left(M_{j}^{\prime \perp} \oplus M_{j+1}^{\prime}\right)=M_{j+1}^{\prime} .
$$

It follows that for any $j \in \mathbb{N}, 1 \leq j \leq n ; M_{j+1}^{\prime \perp}=M_{j}^{\prime \perp} \oplus N_{j}^{\prime}$ It is easy to see that $\operatorname{codim} M_{j}^{\prime}=$ $\sum_{1 \leq i \leq j} \operatorname{dim} N_{i}^{\prime}<\infty$.

Remark 5 If $\left(P_{i}\right)$ is a sequence of mutually orthogonal projections in $H$, then $\sum_{1 \leq i \leq n} P_{i}$ converges to $P$, which is an orthogonal projection (see [13]).

Suppose first that the sequence $\left(\mu_{n}\right)$ is finite, $1 \leq j \leq n$. We put $H_{1}=\bigcap_{1 \leq j \leq n} M_{j}$ and $K_{2}=\sum_{1 \leq j \leq n}\left(\mu_{j}^{\prime}-\mu_{j}\right) P_{j}$. It is obvious that $K_{2}$ is normal.

Lemma 7 If $\mu \in \rho_{e}(B)$, then $N\left(B+K_{2}-\mu I\right) \subseteq N(B-\mu I) \cap H_{1}$.

Proof First show by induction that if $u \in N\left(B+K_{2}-\mu I\right)$ then $u \in H_{1}$. Let $u \in N\left(B+K_{2}-\mu I\right)$, then $P_{1}^{\prime}=0$. Indeed

$$
\left(B+K_{2}-\mu I\right)\left(I-P_{1}^{\prime}\right) u+\left(B+K_{2}-\mu I\right) P_{1}^{\prime} u=\left(B+K_{2}-\mu I\right)=0 .
$$

But

$$
K_{2} P_{1}^{\prime} u=\left[\sum_{1 \leq k \leq n}\left(\mu_{k}^{\prime}-\mu_{k}\right) P_{k}^{\prime}\right] P_{1}^{\prime} u=\left(\mu_{1}^{\prime}-\mu_{1}\right) P_{1}^{\prime} u .
$$

Hence

$$
\begin{aligned}
0= & \left(B+K_{2}-\mu I\right)\left(I-P_{1}^{\prime}\right) u+B-\mu_{1} I+\left(\mu_{1}^{\prime}-\mu_{1}\right) I P_{1}^{\prime} u \\
= & \left(B+K_{2}-\mu I\right)\left(I-P_{1}^{\prime}\right) u+B-\mu_{1} I+\left(\mu_{1}^{\prime}-\mu_{1}\right)\left(I-P_{1}\right) P_{1}^{\prime} u \\
& +B-\mu_{1} I+\left(\mu_{1}^{\prime}-\mu_{1}\right) I P_{1} P_{1}^{\prime} u .
\end{aligned}
$$

Now we account for the last two terms of the amount in $M_{1}$ (because $K_{2}\left(I-P_{1}^{\prime}\right)=(I-$ $\left.P_{1}^{\prime}\right) K_{2}$ is invariant under $B$ ) and the last part of $N_{1}$ which is invariant under $B$. So as $H=$ $M_{1} \oplus N_{1}$ then $B-\mu_{1} I+\left(\mu_{1}^{\prime}-\mu\right) I P_{1} P_{1}^{\prime} u=0$. The operator $\left(B-\mu_{1} I\right) \mid N_{1}$ is nilpotent; and as 
$\mu_{1}=\mu$, then $B-\mu_{1} I+\left(\mu_{1}^{\prime}-\mu\right) I$ is one-to-one. It follows that $P_{1} P_{1}^{\prime} u=0$. Hence $P_{1}^{\prime} u=0$. Suppose that always $u \in N\left(B+K_{2}-\mu I\right) ; P_{j}^{\prime}=0$ with $j=1,2,3, \ldots, m$. According to Lemma 5, $u \in M_{m}^{\prime} u$, we show $P_{m+1}^{\prime} u=0$. Indeed

$$
\left(B+K_{2}-\mu I\right)\left(I-P_{m+1}^{\prime}\right) u+\left(B+K_{2}-\mu I\right) P_{m+1}^{\prime} u=\left(B+K_{2}-\mu I\right)=0 .
$$

But $K_{2} P_{m+1}^{\prime} u=\left(\mu_{m+1}^{\prime}-\mu_{m+1}\right) P_{m+1}^{\prime} u$, and $\left(I-P_{m+1}^{\prime}\right) \in M_{m+1}^{\prime} \subseteq M_{m+1}$. Hence

$$
\left(B+K_{2}-\mu I\right)\left(I-P_{m+1}^{\prime}\right) u+B-\mu_{m+1} I+\left(\mu_{m+1}^{\prime}-\mu\right) I P_{m+1}^{\prime} u=0 .
$$

But

$$
\left(B+K_{2}-\mu I\right)\left(I-P_{m+1}^{\prime}\right) u=(B-\mu I)\left(I-P_{m+1}^{\prime}\right) u+K_{2}\left(I-P_{m+1}^{\prime} u\right) \in M_{m+1} .
$$

Because $M_{m+1}$ is invariant under $B-\mu I$ and $M_{m+1}^{\prime}$ is invariant under $K_{2}$,

$$
\begin{aligned}
B- & \mu_{m+1} I+\left(\mu_{m+1}^{\prime}-\mu\right) I P_{m+1}^{\prime} u \\
= & B-\mu_{m+1} I+\left(\mu_{m+1}^{\prime}-\mu\right) I\left(I-P_{m+1}\right) P_{m+1} u \\
& +B-\mu_{m+1} I+\left(\mu_{m+1}^{\prime}-\mu\right) I P_{m+1} P_{m+1}^{\prime} u .
\end{aligned}
$$

The first term is in $M_{m+1}$ while the second is in $N_{m+1}$ (same reasons as before). So as $H=$ $M_{m+1} \oplus N_{m+1}$, and as above, we deduce from (2.1) that $B-\mu_{m+1} I+\left(\mu_{m+1}^{\prime}-\mu\right) I P_{m+1} P_{m+1}^{\prime} u=0$ then $P_{m+1} P_{m+1}^{\prime} u=0$. Hence $P_{m+1} u \in M_{m+1}$. So by Lemma 6 and using the induction hypothesis $u \in M_{m+1}$ and therefore $P_{m+1}^{\prime} u=0$, which states that $u \in H_{1}$ and $K_{2} u=0$. If the sequence $\mu_{n}$ is infinite, we set $H_{1}=\bigcap_{1 \leq j \leq \infty} M_{j}$ and $K_{2} \sum_{1 \leq j \leq \infty}\left(\mu_{j}^{\prime}-\mu_{j}\right) P_{j}^{\prime}$, the previous proposal remains valid because the series defining $K_{2}$ is normally convergent. Indeed $\lim _{j \rightarrow \infty}\left(\mu_{j}^{\prime}-\mu_{j}\right)=0 \Leftrightarrow(\forall \varepsilon>0)(\exists N(\varepsilon)>0)\left(\forall j \geq N(\varepsilon) \Rightarrow\left|\mu_{j}^{\prime}-\mu_{j}\right|<\varepsilon\right)$. Let $u \in H$ and $n \geq N(\varepsilon)$ then

$$
\begin{aligned}
\left\|\sum_{k \geq n}\left(\mu_{k}^{\prime}-\mu_{k}\right) P_{k}^{\prime} u\right\|^{2} & =\left\langle\sum_{k \geq n}\left(\mu_{k}^{\prime}-\mu_{k}\right) P_{k}^{\prime} u, \sum_{j \geq n}\left(\mu_{j}^{\prime}-\mu_{j}\right) P_{j}^{\prime} u\right\rangle \\
& =\left\langle\sum_{k \geq n} \sum_{j \geq n}\left(\mu_{k}^{\prime}-\mu_{k}\right) \overline{\left(\mu_{j}^{\prime}-\mu_{j}\right)} P_{j}^{\prime} P_{k}^{\prime} u, u\right\rangle \\
& =\sum_{k \geq n}\left|\mu_{k}^{\prime}-\mu_{k}\right|^{2}\left\langle P_{k}^{\prime} u, P_{k}^{\prime} u\right\rangle \leq \varepsilon^{2}\left\|\sum_{k \geq n} P_{k}^{\prime} u\right\|^{2} \\
& \leq \varepsilon^{2}\left\|P^{\prime} u\right\|^{2} \leq \varepsilon^{2}\|u\|^{2} .
\end{aligned}
$$

Therefore $\left\|\sum_{k \geq n}\left(\mu_{k}^{\prime}-\mu_{k}\right) P_{k}^{\prime} u\right\| \leq \varepsilon$, then $\sum_{1 \leq j \leq \infty}\left(\mu_{j}^{\prime}-\mu_{j}\right) P_{j}^{\prime}$ is normally convergent. The rest of the proof is similar to the case of finite $\mu_{j}$.

Lemma 8 If $\mu \in \rho_{e}(B)$, then $H_{1}^{\perp} \subseteq R\left(B+K_{2}-\mu I\right)+H_{1}$.

Proof (A) First prove that for any $j \in \mathbb{N}$, if $\mu_{j} \in N_{j}^{\prime}$ then there exists one and only one $w_{j} \in N_{j}^{\prime}$ such that

$$
P_{j}^{\prime}\left(B+K_{2}-\mu I\right) w_{j}=u_{j}
$$


Uniqueness. Suppose that $w_{j}$ and $w_{j}^{\prime}$ satisfy the requirement. Then $w_{j}-w_{j}^{\prime} \in N\left(B+K_{2}-\right.$ $\mu I) \subseteq H_{1}$ (see Lemma 6), hence $w_{j}-w_{j}^{\prime} \in N_{j}^{\prime} \cap H_{1}=\{0\}$.

Existence. If $u_{j} \in N_{j}^{\prime}$ then $u_{j}=x_{j}+y_{j}$ with $x_{j} \in N_{j}, y_{j} \in M_{j}^{\prime}$ and $u_{j}=P_{j}^{\prime} x_{j}$. We have $B-\mu I+$ $\left(\mu_{j}^{\prime}-\mu_{j}\right) I=B-\mu_{j} I+\left(\mu_{j}^{\prime}-\mu\right) I$ and as $\mu_{j}^{\prime} \neq \mu$, there is $v_{j} \in N_{j}$ such that $x_{j}=\left[B-\mu I+\left(\mu_{j}^{\prime}-\right.\right.$ $\left.\left.\mu_{j}\right) I\right] v_{j}$ then

$$
\begin{aligned}
u_{j} & =P_{j}^{\prime}\left[B-\mu I+\left(\mu_{j}^{\prime}-\mu_{j}\right) I\right]\left[P_{j}^{\prime} v_{j}+\left(I-P_{j}^{\prime}\right) v_{j}\right] \\
& =P_{j}^{\prime}\left[B-\mu I+K_{2}\right] P_{j}^{\prime} v_{j}+P_{j}^{\prime}\left[B-\mu I+\left(\mu_{j}^{\prime}-\mu_{j}\right) I\right]\left(I-P_{j}^{\prime}\right) v_{j} .
\end{aligned}
$$

$\operatorname{But}\left(I-P_{j}^{\prime}\right) v j \in M_{j}^{\prime}$. Hence $\left[B-\mu I+\left(\mu_{j}^{\prime}-\mu_{j}\right) I\right]\left(I-P_{j}^{\prime}\right) v_{j} \in M_{j}^{\prime}$ and therefore $P_{j}^{\prime}\left[B-\mu I+\left(\mu_{j}^{\prime}-\right.\right.$ $\left.\left.\mu_{j}\right) I\right]\left(I-P_{j}^{\prime}\right) v_{j}=0$. We take $w_{j}=P_{j}^{\prime} v_{j} \in N_{j}^{\prime}$.

(B) Let $Q=\sum_{j} P_{j}^{\prime}, u_{j}=P_{j}^{\prime} u$, and $u \in H_{1}^{\perp}$. We show that there is $w \in H_{1}^{\perp}$ such that

$$
u=Q\left(B+K_{2}-\mu I\right) w
$$

It follows from the existence of $w_{1} \in N_{1}^{\prime}$ that $P_{1}^{\prime}\left(B+K_{2}-\mu I\right) w_{1}=u_{1}$. Suppose we know $w_{1}, w_{2}, w_{3}, \ldots, w_{n}, u_{n+1}-\sum_{1 \leq j \leq n} P_{n+1}^{\prime}\left(B+K_{2}-\mu I\right) w_{j} \in N_{n+1}^{\prime}$, then there is $w_{n+1} \in N_{n+1}^{\prime}$ such that

$$
P_{n+1}^{\prime}\left(B+K_{2}-\mu I\right) w_{n+1}=u_{n+1}-\sum_{1 \leq j \leq n} P_{n+1}^{\prime}\left(B+K_{2}-\mu I\right) w_{j},
$$

and therefore

$$
u_{n+1}=\sum_{1 \leq j \leq n} P_{n+1}^{\prime}\left(B+K_{2}-\mu I\right) w_{j}
$$

Let $w=\sum_{j} w_{j}$. Then

$$
\begin{aligned}
Q\left(B+K_{2}-\mu I\right) w & =\sum_{j} P_{j}^{\prime}\left(B+K_{2}-\mu I\right) \sum_{i} w_{i} \\
& =\sum_{j} \sum_{i} P_{j}^{\prime}\left(B+K_{2}-\mu I\right) w_{i} \\
& =\sum_{j} \sum_{i} P_{j}^{\prime}\left[B-\mu I+\left(\mu_{i}^{\prime}-\mu_{i}\right) I\right] w_{i} .
\end{aligned}
$$

But $w_{i} \in M_{i-1}^{\prime},\left[B-\mu I+\left(\mu_{i}^{\prime}-\mu_{i}\right) I\right] w_{i} \in M_{i-1}^{\prime}$ and if $j<i$ we have $P_{j}^{\prime}\left(M_{i-1}^{\prime}\right)=0$. Then

$$
Q\left(B+K_{2}-\mu I\right) w=\sum_{j} \sum_{1 \leq i \leq j} P_{j}^{\prime}\left(B+K_{2}-\mu I\right) w_{i}
$$

Using this we find $Q\left(B+K_{2}-\mu I\right) w=\sum_{j} u_{j}=u$; then $u=\left(B+K_{2}-\mu I\right) w-(I-Q)\left(B+K_{2}-\right.$ $\mu I) w$. Hence $u \in R\left(B+K_{2}-\mu I\right)+H_{1}$. This completes the proof of the lemma.

Lemma 9 Let $B \in L(H)$ such that $\rho_{e}(B) \cap \operatorname{reg}(B) \subseteq M_{e}(B)$. Then there is a compact operator $K_{2} \in K(H)$ such that

$$
\rho_{e}(B)=\rho_{e}\left(B+K_{2}\right) \subseteq M_{e}\left(B+K_{2}\right) .
$$


Proof We show that

$$
\rho e(B) \subseteq \operatorname{reg}\left(B+K_{2}\right)
$$

Let $\mu \in \rho_{e}(B)$ and $u \in N\left(B+K_{2}-\mu I\right)$. Then $u \in N(B-\mu I) \cap H_{1}$. But $(B-\mu I) \mid H_{1}$ is regular because for any $j \in \mathbb{N},(B-\mu I) \mid M_{j}$ is also regular. Hence for any $j \in \mathbb{N}, u \in R\left[(B-\mu I)^{j} \mid H_{1}\right] \subseteq$ $R\left[\left(B+K_{2}-\mu I\right)^{j}\right]$. As further $B+K_{2}-\mu I$ is a compact perturbation of the semi-Fredholm operator $B-\mu I$, it is itself semi-Fredholm and hence $R\left(B+K_{2}-\mu I\right)$ is a closed subspace, which completes the demonstration We now show that $\operatorname{reg}\left(B+K_{2}\right) \subseteq M_{e}\left(B+K_{2}\right)$. Let $\rho e(B)=\bigcup_{k} C_{k}=\operatorname{reg}\left(B+K_{2}\right)$ where $C_{k}$ is the $n$th $k$-connected component of $\rho_{e}(B)$.

For any $k \in \mathbb{N}^{*}, C_{k} \cap \operatorname{reg}(B)=\emptyset$. Let $\mu \in C_{k} \cap \operatorname{reg}(B)=C_{k} \cap M_{e}(B)$.

Two cases are possible.

First case. $B-\mu I$ is one-to-one and therefore $N\left(B+K_{2}-\mu I\right) \subseteq N(B-\mu I)=0 \Rightarrow B+K_{2}-$ $\mu I$ is one-to-one, hence $C_{k} \subseteq M_{e}\left(B+K_{2}\right)$.

Second case. $B-\mu I$ is onto and therefore $H_{1} \subseteq R(B-\mu I)$. For any $u \in H_{1}$, there is $v$ such that $(B-\mu I) v=u$;

$$
(B-\mu I) Q v=(B-\mu I) v-(B-\mu I)(I-Q) v
$$

$(B-\mu I) v \in H_{1},(I-Q) v \in H_{1} \Rightarrow(B-\mu I)(I-Q) v \in H_{1}, Q v=\sum_{j} P_{j}^{\prime} v=v_{j}$ where $v_{j}=\sum_{j} P_{j}^{\prime} \nu$. We have

$$
(B-\mu I) Q v=\sum_{j}(B-\mu I) v_{j}=\sum_{j}(B-\mu I) P_{j} v_{j}+\sum_{j}(B-\mu I)\left(I-P_{j}\right) v_{j},
$$

where

$$
\sum_{j}(B-\mu I) P_{j} v_{j} \in N_{j} \quad \text { and } \quad \sum_{j}(B-\mu I)\left(I-P_{j}\right) v_{j} \in M_{j}^{\prime}
$$

because $v_{j} \in N_{j}^{\prime} \subseteq M_{j-1}^{\prime} \Rightarrow\left(I-P_{j}\right) v_{j} \in M_{j}^{\prime}$. We have

$$
(B-\mu I) P_{1} v_{1}=(B-\mu I) Q v-\sum_{j>1}(B-\mu I) P_{j} v_{j}-\sum_{j}(B-\mu I)\left(I-P_{j}\right) v_{j} \in M_{1} .
$$

Hence $(B-\mu I) P_{1} v_{1}=0$ because

$$
(B-\mu I) P_{1} v_{1} \in M_{1} \cap N_{1}=0 .
$$

As $\mu \in \operatorname{reg}(B) \Rightarrow \mu_{1}=\mu$ and $B-\mu I=B-\mu_{1}+\left(\mu_{1}-\mu\right) I$. We can see that $P_{1} v_{1}=0$, hence $v_{1} \in M_{1} \cap N_{1}=0 \Rightarrow v_{1}=0$. Suppose we have shown that $v_{1}=v_{2}=v_{3}=\cdots=v_{n}=0$ and we demonstrate that $v_{n+1}=0$. As previously $(B-\mu I) P_{n+1} v_{n+1} \in M_{n+1}^{\prime}$ hence $(B-\mu I) P_{n+1} v_{n+1}=$ 0 , and as $\mu \in \operatorname{reg}(B) \Rightarrow \mu=\mu_{n+1} \Rightarrow P_{n+1} v_{n+1}=0$, then $v_{n+1} \in M_{n+1} \cap N_{n+1}^{\prime}=0 \Rightarrow v_{n+1}=0$. It follows that $(B-\mu I) Q v=0$ hence $u=(B-\mu I) w$ with $w=(I-Q) v \in H_{1}$; therefore $u=$ $\left(B+K_{2}-\mu I\right) w \Rightarrow u \in R\left(B+K_{2}-\mu I\right)$. Hence $H_{1} \subseteq R\left(B+K_{2}-\mu I\right)$ and (by Lemma 7) we have $H_{1}^{\perp} \subseteq R\left(B+K_{2}-\mu I\right)$; hence $H \subseteq R\left(B+K_{2}-\mu I\right)$, which implies that $B+K_{2}-\mu I$ is onto and $C_{k} \subseteq M_{e}(B+K)_{2}$ and therefore $\rho_{e}(B)=\sum_{k} C_{k} \subseteq M_{e}\left(B+K_{2}\right)$, which is equivalent to $\rho_{e}(B)=M_{e}\left(B+K_{2}\right)$. 
Theorem 7 Let $A \in L(H)$. There is a compact operator $K \in K(H)$ such that

$$
\rho_{e}(A)=M_{e}(A+K)
$$

Proof This is an immediate consequence of Lemma 5 and Lemma 9; simply take $K=K_{1}+$ $K_{2}$.

Corollary 1 Let $A$ be a Riesz operator. Then there exists a normal compact operator $K$ such that $(A-K) \in Q N(H)$.

Proof $A$ satisfies the hypothesis of Lemma 9 and also $\rho_{e}(A)=\mathbb{C} \backslash 0$ and $\operatorname{reg}(A)=\mathbb{C} \backslash \sigma(A)=$ $\rho(A)$

$\rho_{e}(A) \cap \operatorname{reg}(A)=\rho(A)=M_{e}(A)$. Hence there is a compact operator $K$ such that $\rho(A-K)=$ $M_{e}(A-K)=\rho_{e}(A)=\mathbb{C} \backslash 0 \Rightarrow \sigma(A-K)=0 \Rightarrow(A-K) \in Q N(H)$. We find the result of [14].

Remark 6 The result of Stampfli is a special case of Theorem 7 because if $\mu \in \Phi_{0}(A)$, then $\mu \in \rho_{e}(A) \subseteq M_{e}(A+K)$, hence $A+K-\mu I$ is one-to-one or onto. If $A+K-\mu I$ is one-toone (resp. onto), then $N(A+K-\mu I)=0$ (resp. $\left.N\left(A^{*}+K^{*}-\mu I\right)=0\right)$ and $\operatorname{ind}(A+K-\mu I)=$ $\operatorname{ind}(A-\mu I)=0 N\left(A^{*}+K^{*}-\mu I\right)=0(\operatorname{resp} . N(A+K-\mu I)=0)$ and therefore $A+K-\mu I$ is one-to-one and onto. Hence $\mu \in \rho(A+K) \Rightarrow \Phi_{0}(A)=\rho(A+K)$.

Remark 7 In 1966, West has shown in [14] that for any Riesz operator $A \in L(H)$ such that $\rho_{e}(A) \subseteq \mathbb{C} \backslash 0$ there is a compact operator $K \in K(H)$ and $Q \in Q N(H)$ such that $A=K+Q$. We find this result as a special case of our result (see Corollary 1 ).

\section{Conclusion}

After the famous result of Stampfli, for all bounded operator $A$ the Weyl spectrum is $\sigma_{W}(A)=\bigcap \sigma(A+K)$ where the intersection is taken over all closed ideal compact operators. In this work, we prove the possibility to extend this result by the use of Kato's decomposition to the set of semi-Fredholm operators.

Competing interests

The authors declare that they have no competing interests.

Authors' contributions

The authors contributed equally to the writing of this paper, and they read and approved the final manuscript.

\section{Acknowledgements}

The authors are grateful to the anonymous referees who have contributed to improve the quality of this paper.

Received: 25 September 2015 Accepted: 3 February 2016 Published online: 12 February 2016

\section{References}

1. Schechter, M: Invariance of the essential spectrum. Bull. Am. Math. Soc. 71, 365-367 (1965)

2. Stampfli, JG: Compact perturbation, normal eigenvalues and a problem of Salinas. J. Lond. Math. Soc. (2) 9, 165-175 (1974)

3. Apostol, C: The correction by compact perturbation of the singular behaviour of operators. Rev. Roum. Math. Pures Appl. 21(2), 155-175 (1976)

4. Herrero, DA: Approximation of Hilbert Space Operators, vol. 1. Research Notes in Mathematics, vol. 72. Pitman, London (1982)

5. Cordes, HO, Labrousse, J-P: The invariance of the index in the metric space of closed operators. J. Math. Mech. 12(5), 693-720 (1963)

6. Kato, T: Perturbation theory for nullity, deficiency and other quantities of linear operators. J. Anal. Math. 6, 261-322 (1958) 
7. Labrousse, J-P: Les opérateurs quasi-Fredholm: une généralisation des opérateurs semi-Fredholm. Rend. Circ. Mat. Palermo 29, 161-258 (1980)

8. Mbekhta, M: Décomposition de Kato généralisée. C. R. Acad. Sci. Paris, Sér. I Math. 303, 979-982 (1986)

9. Goldberg, S: Unbounded Linear Operators. McGraw-Hill, New York (1966)

10. Nagy, BS: On the stability of the index of unbounded linear transformations. Acta Math. Acad. Sci. Hung. 3, 49-52 (1952)

11. Schwartz, L: Analyse Hilbertienne: Collection Méthodes. Hermann, Paris (1979)

12. Dunford, N, Schwartz, JT: Linear Operators, parts I, II. Interscience, New York (1988)

13. Kato, T: Perturbation Theory of Linear Operators. Springer, New York (1966)

14. West, TT: The decomposition of Riesz operators. Proc. Lond. Math. Soc. 16, 737-752 (1966)

\section{Submit your manuscript to a SpringerOpen ${ }^{\circ}$} journal and benefit from:

- Convenient online submission

Rigorous peer review

- Immediate publication on acceptance

- Open access: articles freely available online

- High visibility within the field

- Retaining the copyright to your article 